\title{
Comprehensive Radionuclide Esophagogastrointestinal Transit Study: Methodology, Reference Values, and Initial Clinical Experience
}

\author{
Alexander J. Antoniou ${ }^{1}$, Shreya Raja ${ }^{2}$, Riham El-Khouli ${ }^{1}$, Esther Mena ${ }^{1}$, Martin A. Lodge ${ }^{1}$, Richard L. Wahl ${ }^{1}$, \\ John O. Clarke ${ }^{2}$, Pankaj Pasricha ${ }^{2}$, and Harvey A. Ziessman ${ }^{1}$ \\ ${ }^{1}$ Division of Nuclear Medicine, Russell H. Morgan Department of Radiology and Radiological Sciences; and ${ }^{2}$ Division of \\ Gastroenterology and Hepatology, Department of Internal Medicine, Johns Hopkins University, Baltimore, Maryland
}

\begin{abstract}
A radionuclide methodology and reference values have been developed for a single gastrointestinal transit study including esophageal transit, liquid and solid gastric emptying, and small- and largebowel transit, using ${ }^{111}$ In-diethylenetriaminepentaacetic acid (DTPA) with the standardized ${ }^{99 \mathrm{~m} T c-l a b e l e d}$ solid meal. Methods: Eighteen healthy subjects and 18 patients were investigated. The esophageal transit study was performed with $3.7 \mathrm{MBq}(0.1 \mathrm{mCi})$ of ${ }^{111} / \mathrm{n}$-DTPA in $15 \mathrm{~mL}$ of water. A liquid-only 30-min gastric-emptying study followed, with ingestion of $3.7 \mathrm{MBq}(0.1 \mathrm{mCi})$ of ${ }^{111} \mathrm{In}$-DTPA in $300 \mathrm{~mL}$ of water. Then, a simultaneous solid-liquid emptying study was acquired after ingestion of a solid ${ }^{99 m} \mathrm{Tc}$-sulfur colloid-labeled meal and $7.4 \mathrm{MBq}$ $(0.2 \mathrm{mCi})$ of ${ }^{111} \mathrm{In}-\mathrm{DTPA}$ in $120 \mathrm{~mL}$ of water. Images were acquired intermittently for $4 \mathrm{~h}$. Additional ${ }^{111} \mathrm{In}$ images were acquired at 5 and 6 $\mathrm{h}$ to measure small-bowel transit, and at 24,48 , and $72 \mathrm{~h}$ for largebowel transit. Results: Reference values were determined for esophageal transit (transit time, percentage emptying at $10 \mathrm{~s}$ ), liquid-only gastric emptying (emptying half-time), liquid and solid emptying in a dual-phase solid-liquid study (emptying half-time and percentage emptying at 1, 2, 3, and $4 \mathrm{~h}$ ), small-bowel transit index (percentage transit to ileocecal valve at $6 \mathrm{~h}$ ), and colonic transit (geometric center and percentage colonic emptying) at 24,48 , and $72 \mathrm{~h}$. Results from the first 18 patients found abnormal transit in $72 \%$ (13/18); clinical management changed in $61 \%$ (11/18). Conclusion: We have developed a radionuclide methodology and derived reference values for a comprehensive gastrointestinal transit study using ${ }^{111} \mathrm{In}$-DTPA with the standardized ${ }^{99 m T c-l a b e l e d ~ s o l i d ~ m e a l . ~ O u r ~ i n i t i a l ~ c l i n i c a l ~ e x p e r i-~}$ ence suggests clinical value.
\end{abstract}

Key Words: intestinal transit; esophageal scintigraphy; gastric emptying; gastrointestinal scintigraphy

J Nucl Med 2015; 56:721-727

DOI: 10.2967/jnumed.114.152074

\section{$\mathbf{P}$} ety, postprandial abdominal discomfort, bloating, fullness, and nausea are referred for radionuclide gastric-emptying studies for suspected gastroparesis (delayed gastric emptying), a functional disorder usually without

\footnotetext{
Received Nov. 25, 2014; revision accepted Jan. 14, 2015.

For correspondence or reprints contact: Harvey A. Ziessman, $601 \mathrm{~N}$. Caroline St., JHOC 3231, Baltimore, MD 21287.

E-mail: hziessm1@jhmi.edu.

Published online Mar. 12, 2015.

COPYRIGHT (C 2015 by the Society of Nuclear Medicine and Molecular Imaging, Inc.
}

anatomic correlation. However, until recently, gastroparesis was diagnosed by the study in only $20 \%-40 \%$ of patients (1).

Recent progress has been made in improving the diagnostic yield of the test. Extending the study from 2 to $4 \mathrm{~h}$ has increased the diagnosis of gastroparesis by a third (2-4). Liquid gastricemptying studies can further increase detection of gastroparesis by another third (5-7). Still, in many patients the cause of their symptoms goes undiagnosed.

Gastrointestinal symptoms are often nonspecific as to the organ of origin. Dyspeptic symptoms are common in both gastric and small-bowel dysmotility and are reported with esophageal dysmotility. Although constipation and diarrhea predominate in largebowel transit disease, these patients often have symptoms that overlap with those of the upper gastrointestinal tract. Transit abnormalities may occur in more than one gastrointestinal organ. If we could routinely investigate patients for small- and large-bowel dysmotility in addition to gastric emptying, we might be able diagnose additional patients with functional gastrointestinal disease.

Conventional intestinal transit studies such as the hydrogen breath test, radiopaque markers, and wireless motility capsules can be invasive, costly, have a high radiation dose, or be of limited reliability and nonphysiologic $(8,9)$. Radionuclide methods studying intestinal transit have been published, but few centers offer them and there is no standard methodology or generally applicable reference values, although a practice guideline was recently published (10).

The purpose of this investigation was to develop a physiologic radionuclide methodology and establish reference values for a comprehensive gastrointestinal transit clinical study, including esophageal transit, liquid and solid gastric emptying, and smalland large-bowel transit, using ${ }^{111}$ In-diethylenetriaminepentaacetic acid (DTPA) in conjunction with the consensus-recommended standardized ${ }^{99 \mathrm{~m} T c}$-labeled solid meal $(11-13)$ and to present our initial clinical experience.

\section{MATERIALS AND METHODS}

\section{Study Population}

Eighteen healthy subjects ( 10 men, 8 women; age range, $20-57$ y; mean \pm SD, $33.6 \pm 11.9$ y) were enrolled. The Mayo Clinic Research Questionnaire was used to interview and screen them. The subjects had no history of gastrointestinal disorders or prior surgery, were on no medications, and did not smoke or abuse alcohol. The investigational protocol was approved by the Johns Hopkins University Institutional Review Board. Written informed consent was obtained. The initial 18 consecutive patients (5 men, 13 women; age range, 20-72 y; mean, $52.6 \pm 16.4$ ) were referred because of a variety of gastrointestinal symptoms (Table 1). 
TABLE 1

Clinical Patient Results

\begin{tabular}{|c|c|c|c|c|c|c|c|c|c|c|c|c|c|c|}
\hline \multirow[b]{2}{*}{ Patient } & \multirow[b]{2}{*}{ Age $(y)$} & \multirow[b]{2}{*}{ Sex } & \multirow{2}{*}{$\begin{array}{c}\text { Previous } \\
\text { abnormal } \\
\text { motility } \\
\text { results }^{\star}\end{array}$} & \multicolumn{3}{|c|}{$\begin{array}{l}\text { Suspected } \\
\text { dysmotility }\end{array}$} & \multicolumn{6}{|c|}{ Scintigraphic findings } & \multirow{2}{*}{$\begin{array}{l}\text { Change } \\
\text { in } \\
\text { diagnosis }\end{array}$} & \multirow{2}{*}{$\begin{array}{c}\text { Change in } \\
\text { management }\end{array}$} \\
\hline & & & & $E$ & $\mathrm{G} / \mathrm{SB}$ & $\mathrm{C}$ & $\mathrm{E}$ & $\mathrm{GL}$ & GLc & GSc & SB & $\mathrm{C}$ & & \\
\hline 1 & 26 & $\mathrm{~F}$ & Yes - E & + & + & + & & & & & & & No & Yes \\
\hline 2 & 47 & M & No & & + & + & $\mathrm{D}$ & & & & & $\mathrm{D}$ & Yes & No \\
\hline 3 & 74 & M & No & & + & & & & & & & & No & No \\
\hline 4 & 62 & $\mathrm{~F}$ & Yes - E & + & + & + & & & & & & $\mathrm{D}$ & Yes & Yes \\
\hline 5 & 72 & $\mathrm{~F}$ & No & & + & + & & & $\mathrm{D}$ & & $\mathrm{D}$ & 1 & Yes & Yes \\
\hline 6 & 46 & $M$ & Yes - G & & + & + & & & & & & $\mathrm{D}$ & Yes & Yes \\
\hline 7 & 64 & M & No & & & + & & & & & & $\mathrm{D}$ & Yes & No \\
\hline 8 & 55 & $\mathrm{~F}$ & Yes - SB & & + & + & & & & & & $D$ & Yes & Yes \\
\hline 9 & 51 & $\mathrm{~F}$ & Yes - G, C & & + & + & & D & D & D & & D & No & No \\
\hline 10 & 67 & $M$ & No & & + & + & & & & & & & No & No \\
\hline 11 & 22 & $\mathrm{~F}$ & No & + & + & + & & & & & & & No & Yes \\
\hline 12 & 65 & $\mathrm{~F}$ & Yes - G, C & + & + & + & & $D$ & & $D$ & & & Yes & No \\
\hline 13 & 39 & $F$ & No & & + & & & & & & & $\mathrm{R}$ & Yes & No \\
\hline 14 & 62 & $\mathrm{~F}$ & Yes - G & & + & + & D & & & & & D & Yes & Yes \\
\hline 15 & 52 & $\mathrm{~F}$ & No & & + & + & & & & & & & No & Yes \\
\hline 16 & 72 & $\mathrm{~F}$ & Yes - G & + & + & + & & $D$ & $\mathrm{D}$ & & $D$ & $D$ & Yes & Yes \\
\hline 17 & 20 & $\mathrm{~F}$ & No & + & + & + & & & & & & D & Yes & Yes \\
\hline 18 & 50 & $\mathrm{~F}$ & Yes - E, G & + & + & + & & & & & $D$ & $\mathrm{R}$ & Yes & Yes \\
\hline
\end{tabular}

*Previous abnormal testing included E (manometry), G (scintigraphy), SB (small-bowel follow-through; hydrogen breath test), and C (Sitz markers; anorectal manometry).

$\mathrm{E}=$ esophageal; $\mathrm{G}=$ gastric; $\mathrm{SB}=$ small bowel; $\mathrm{C}=$ colon; $\mathrm{GL}=$ gastric liquid only; $\mathrm{GLc}=$ gastric liquid combined; GSc $=$ gastric solid combined; D = delayed transit; I = indeterminate (significantly delayed SB transit persistent until $72 \mathrm{~h}$ ); $\mathrm{R}=\mathrm{rapid}$ transit.

\section{Methodology}

All subjects and patients were instructed to fast overnight before the study. Patients took medications prescribed by their referring physicians.

A dual-head large-field-of-view $\gamma$ camera with medium-energy collimators was used. Images were acquired with a $20 \%$ window around the photopeaks of ${ }^{99 \mathrm{~m}} \mathrm{Tc}(140 \mathrm{keV})$ and ${ }^{111} \mathrm{In}(171,245 \mathrm{keV})$.

Esophageal Transit. Two practice swallows (15 mL of water) with a 15-s delay between swallows were followed by a third ( ${ }^{111}$ In-DTPA, 3.7 $\mathrm{MBq}[0.1 \mathrm{mCi}]$ in $15 \mathrm{~mL}$ of water). Data acquisition began before the third swallow, and 60 frames of $0.5 \mathrm{~s}$ each $(64 \times 64)$ were acquired posteriorly with the subject standing. The field of view included the mouth through the upper stomach.

To process, a region of interest (ROI) was drawn around the esophagus from the level of the cricoid to the lower esophageal sphincter using Xeleris esophageal swallow software (GE Healthcare). Both esophageal transit time (time for activity to decrease to $10 \%$ of peak) and percentage esophageal emptying (\% activity decrease from peak over $10 \mathrm{~s}$ ) were calculated.

Liquid-Only Gastric Emptying. A 30-min liquid-only study was acquired (1-min frames, $64 \times 64)$ after ingestion of $300 \mathrm{~mL}$ of water with ${ }^{111} \mathrm{In}-\mathrm{DTPA}$ (3.7 MBq [0.1 mCi]). Patients were imaged while semiupright (45\%), lying supine on a hospital gurney, with the $\gamma$ camera placed in the left anterior oblique position. To process, a ROI was drawn for the stomach. The half-time of emptying and a fitted exponential half-time were determined.

Combined Liquid-Solid Gastric Emptying. Subjects ingested the standardized ${ }^{99 \mathrm{~m} T c}$-sulfur colloid (7.4 MBq [0.2 mCi]) egg-substitute solid meal (113 g [4 oz] of Optimum Choice; Sysco Corp.), 2 slices of toast, $30 \mathrm{~g}$ of strawberry jam (11-13), and $120 \mathrm{~mL}$ of water mixed with ${ }^{111} \mathrm{In}$-DTPA (7.4 MBq [0.2 $\left.\left.\mathrm{mCi}\right]\right)$. Anterior and posterior simultaneous dual-isotope images were acquired at 0.0, 0.5, 1.0, 1.5, 2, 3, and $4 \mathrm{~h}$. Processing used gastric-emptying software (GE Healthcare) with decay and geometric mean attenuation correction.

To process the liquid portion of the study, the half-emptying time, fitted exponential half-time, and percentage gastric emptying were calculated at each time point. The solid meal was processed similarly, except using a fitted linear half-time.

Patients were instructed not to eat until imaging was concluded on day 1 of the study, but they were allowed to go about normal activities in the clinic vicinity.

Small-Bowel Transit. At 5 and $6 \mathrm{~h}$ after meal ingestion, static anterior and posterior 1 -min images $(128 \times 128)$ were acquired. A small-bowel transit index (SBTI) was determined. To establish counts in the small bowel available to fill the terminal ileum, an ROI was drawn to include the entire abdomen to calculate a decay-corrected geometric mean average of total abdominal counts between 2 and $5 \mathrm{~h}$, corrected for gastric counts. On the 6-h image, an ROI was drawn around the ileocecal valve or cecum and around any colonic activity. The terminal ileum or cecum was located by observing progressive focal accumulation in the right lower quadrant during the first $6 \mathrm{~h}$ of imaging.

To test interobserver bias, 3 residents independently drew the ROI, confirming their choice by observing subsequent transit in the expected large-bowel pattern in the 24-, 48-, and 72-h anterior and posterior images. 
SBTI was calculated by dividing the geometric mean (decaycorrected) percentage ${ }^{111} \mathrm{In}$ activity that had passed into the area at $6 \mathrm{~h}$ by the mean 2- to 5-h abdominal activity. Calculations were facilitated by the use of an Excel worksheet (Microsoft) with decay correction factors.

Large-Bowel Transit. At 24, 48, and 72 h, 5-min images $(128 \times$ $128)$ were acquired in the anterior and posterior views. Geometric mean attenuation and decay correction was performed. Two quantitative methods were performed with the healthy subjects.

First, the geometric center (GC) was determined. The large intestine was manually divided into 7 ROIs (ascending, hepatic flexure, transverse, splenic flexure, descending, rectosigmoid, and excreted activity). The count in each of the 7 segments was multiplied by its corresponding factor (1-7) and then summed. The percentage activity for each segment was calculated as the count in that segment divided by the initial count (average of total abdominal counts between hours 2 and 5). The GC represented a number between 1 and 7 .

Second, percentage colonic emptying at 24,48 , and $72 \mathrm{~h}$ was determined. A ROI was drawn around the entire abdomen at each period. The percentage emptying was calculated using gastric-emptying software by dividing the geometric mean counts at each time point by the amount of initial activity in the colon using the 2- to 5-h average counts, including any gastric counts, and in a separate calculation using the 6-h total counts. The results of the 2 calculations were compared.
Jaszczak Phantom Study. The percentage spillover of one isotope into the window setting of the other was investigated using $42.2 \mathrm{MBq}$ $(1.14 \mathrm{mCi})$ of ${ }^{111} \mathrm{In}$ and $41.8 \mathrm{MBq}(1.13 \mathrm{mCi})$ of ${ }^{99 \mathrm{~m}} \mathrm{Tc}$. ${ }^{99 \mathrm{~m}} \mathrm{Tc}$ spillover into the ${ }^{111} \mathrm{In}$ window was $2.5 \%$ of total ${ }^{111}$ In counts, whereas ${ }^{111} \mathrm{In}$ spillover into the ${ }^{99 \mathrm{~m}} \mathrm{Tc}$ window was $33.1 \%$ of total ${ }^{99 \mathrm{~m}} \mathrm{Tc}$ counts. The $5: 1$ ratio of ${ }^{99 \mathrm{~m}} \mathrm{Tc}$ to ${ }^{111} \mathrm{In}$ in our experimental study would result in a maximum ${ }^{99 \mathrm{~m}} \mathrm{Tc}$ spillover of $12.5 \%$ and a minimum ${ }^{111} \mathrm{In}$ spillover of $6.6 \%$. As ${ }^{99 \mathrm{~m}} \mathrm{Tc}$ decays more quickly than ${ }^{111} \mathrm{In}$, the percentage of spillover from either isotope is expected to range between $6.6 \%$ and $12.5 \%$ during the gastricemptying and small-bowel transit portions of the study.

\section{Statistical Analysis}

Data are presented as mean \pm SD for central tendencies, as median and range when data were skewed, and as frequency and percentage for categoric variables. Between groups, analyses were performed using independent-sample $t$ tests. Statistical significance was set at a 2-tailed $P$ value of 0.05 for all tests. The Shapiro-Wilk test of normality was used. Interobserver analysis was performed using Bland-Altman plots and the Pittman test of difference in variance. All statistical analysis was performed with the JMP statistical package (version 11.0; SAS Institute, Inc.).

The decision on how to best determine reference values was based on the test for normality. Normal was defined as the mean \pm 2 SDs in

TABLE 2

Transit Parameters

\begin{tabular}{|c|c|c|c|c|c|c|c|}
\hline Transit parameter & Mean & Median & SD & Range & $\begin{array}{l}95 \% \text { confidence } \\
\text { interval }\end{array}$ & Skewness & Normality* \\
\hline Esophageal emptying (\%) & 92.3 & 93.5 & 4.8 & $83.1-98.6$ & $89.7,94.8$ & -0.7 & 0.07 \\
\hline Esophageal transit time (s) & 8.8 & 8.5 & 3.2 & $3.5-15.0$ & $7.1,10.5$ & 0.4 & 0.72 \\
\hline Liquid-only half-time (min) & 14.3 & 13.5 & 5.3 & $4.4-22.4$ & $11.5,17.2$ & 0.1 & 0.36 \\
\hline \multicolumn{8}{|l|}{ Liquid-solid combined } \\
\hline Liquid half-time (min) & 38.8 & 33.9 & 15.6 & $19.5-67.8$ & $30.6,47.0$ & 0.6 & 0.04 \\
\hline Liquid $0.5-\mathrm{h}$ percentage emptying & 50.1 & 50.5 & 16.7 & $27.0-82.0$ & $41.8,58.4$ & 0.1 & 0.15 \\
\hline Liquid 1.0-h percentage emptying & 65.2 & 67.0 & 12.9 & $45.0-90.0$ & $58.8,71.6$ & -0.1 & 0.42 \\
\hline Liquid 1.5-h percentage emptying & 78.7 & 77.5 & 9.0 & $74.2-83.1$ & $74.2,83.1$ & 0.4 & 0.29 \\
\hline Liquid 2.0-h percentage emptying & 88.3 & 89.0 & 5.2 & $75.0-95.0$ & $85.7,90.9$ & -1.1 & 0.05 \\
\hline Liquid 3.0-h percentage emptying & 93.6 & 94.5 & 2.5 & $89.0-97.0$ & $92.4,94.8$ & -0.8 & 0.02 \\
\hline Liquid 4.0-h percentage emptying & 95.3 & 96.0 & 2.1 & $90.0-98.0$ & $94.3,96.4$ & -0.9 & 0.13 \\
\hline Solid half-time (min) & 87.7 & 88.3 & 23.8 & $31.3-130.9$ & $75.9,99.6$ & -0.4 & 0.91 \\
\hline Solid $0.5-\mathrm{h}$ percentage emptying & 17.5 & 14.0 & 11.7 & $3.0-51.0$ & $11.7,23.3$ & 1.5 & 0.02 \\
\hline Solid 1.0-h percentage emptying & 31.1 & 32.0 & 17.9 & $7.0-80.0$ & $21.6,40.6$ & 1.1 & 0.09 \\
\hline Solid 1.5-h percentage emptying & 52.5 & 50.0 & 20.0 & $21.0-89.0$ & $42.6,62.5$ & 0.1 & 0.70 \\
\hline Solid 2.0-h percentage emptying & 75.6 & 80.5 & 14.6 & $44.0-97.0$ & $68.4,82.9$ & -0.9 & 0.14 \\
\hline Solid 3.0-h percentage emptying & 94.6 & 95.0 & 3.8 & $83.0-99.0$ & $92.6,96.4$ & -1.7 & 0.01 \\
\hline Solid 4.0-h percentage emptying & 97.7 & 98.5 & 3.1 & $86.0-100.0$ & $96.1,99.2$ & -3.6 & $<0.0001$ \\
\hline SBTI (\%) & 77.3 & 78.5 & 14.0 & $53.0-98.0$ & $70.3,84.2$ & 0.3 & 0.36 \\
\hline Colon GC (24 h) & 4.2 & 3.9 & 1.2 & $2.9-6.7$ & $3.6,4.8$ & 0.9 & 0.04 \\
\hline Colon GC (48 h) & 5.8 & 6.5 & 1.1 & $3.8-6.9$ & $5.2,6.4$ & -0.8 & 0.01 \\
\hline Colon GC (72 h) & 6.5 & 6.7 & 0.6 & $5.2-6.9$ & $6.2,6.8$ & -1.4 & 0.0003 \\
\hline Colon \% emptying (24 h) & 26.6 & 20.5 & 25.2 & $3.0-92.0$ & $14.1,39.1$ & 1.9 & 0.0003 \\
\hline Colon \% emptying (48 h) & 59.4 & 72.0 & 34.9 & $11.0-98.0$ & $41.5,77.4$ & -0.3 & 0.004 \\
\hline Colon \% emptying (72 h) & 77.9 & 87.0 & 22.8 & $31.0-98.0$ & $66.6,89.3$ & -1.2 & 0.001 \\
\hline
\end{tabular}

*Shapiro-Wilk test. 
a parametric sample and as the $95 \%$ confidence interval in a nonparametric sample.

\section{RESULTS}

All 18 subjects completed the study. Table 2 displays the transit results of the healthy subjects; Table 3 the calculated reference values.

\section{Esophageal Transit}

A good swallowed bolus was uniformly observed, and interpretable time-activity curves generated.

\section{Liquid-Only Gastric Emptying}

The time-activity curves usually demonstrated a single-exponential emptying pattern in healthy subjects. Occasionally, overlapping small bowel delayed apparent emptying.

\section{Combined Liquid-Solid Gastric Emptying}

Liquid Emptying. Time-activity curves demonstrated a multiexponential emptying pattern. Normal liquid emptying was slower in the combined study than in the liquid-only study (mean half-time, 38.8 vs.14.3 $\mathrm{min})(P<0.0001)$. Liquid half-time emptying was significantly different without the 0.5 - and 1.5 -h data points $(P=0.0003)$. For clinical patients, we now image only at $0,1,2,3$, and $4 \mathrm{~h}$, using the half-time derived from these time points. Because of the rapid emptying of liquids, the best estimate of liquid emptying was at $1 \mathrm{~h}$.

Solid Emptying. The percentage emptying and half-emptying time at each time point were similar and not significantly different from the published standardized meal data. Thus, for clinical purposes we use the references values of Tougas et al. (11). Unlike liquid emptying, for solid emptying the half-time was not affected by the selection of fewer time points $(P=0.57)$.

\section{Small-Bowel Transit}

An interobserver study (3 residents) showed good correlation for drawing ROIs, with a mean bias of 7.3\%-14.8\% (Pittman test, $P=0.4-0.9$ ). With some experience, our technologists usually accurately draw the ROIs.

TABLE 3

Reference Values

\begin{tabular}{lc}
\hline \multicolumn{1}{c}{ Transit parameter } & Value \\
\hline Esophageal transit time & $<15 \mathrm{~s}$ \\
\hline Esophageal percentage emptying & $>83 \%$ \\
\hline Liquid-only half-time & $<25 \mathrm{~min}$ \\
\hline Liquid with solid half-time & $<74 \mathrm{~min}$ \\
\hline Liquid 1 -h percentage emptying & $>40 \%$ \\
\hline Liquid $2-\mathrm{h}$ percentage emptying & $>78 \%$ \\
\hline Solid 1 -h percentage emptying & $>10 \%$ \\
\hline Solid 2-h percentage emptying & $>40 \%$ \\
\hline Solid 4-h percentage emptying & $>90 \%$ \\
\hline Solid rapid transit $(1 \mathrm{~h})$ & $>70 \%$ \\
\hline SBTI $(6 \mathrm{~h})$ & $>50 \%$ \\
\hline Colon percentage emptying $(24 \mathrm{~h})$ & $>14 \%$ \\
\hline Colon rapid transit $(24$ h) & $>40 \%$ \\
\hline Colon percentage emptying $(48 \mathrm{~h})$ & $>41 \%$ \\
\hline Colon percentage emptying $(72 \mathrm{~h})$ & $>67 \%$ \\
\hline
\end{tabular}

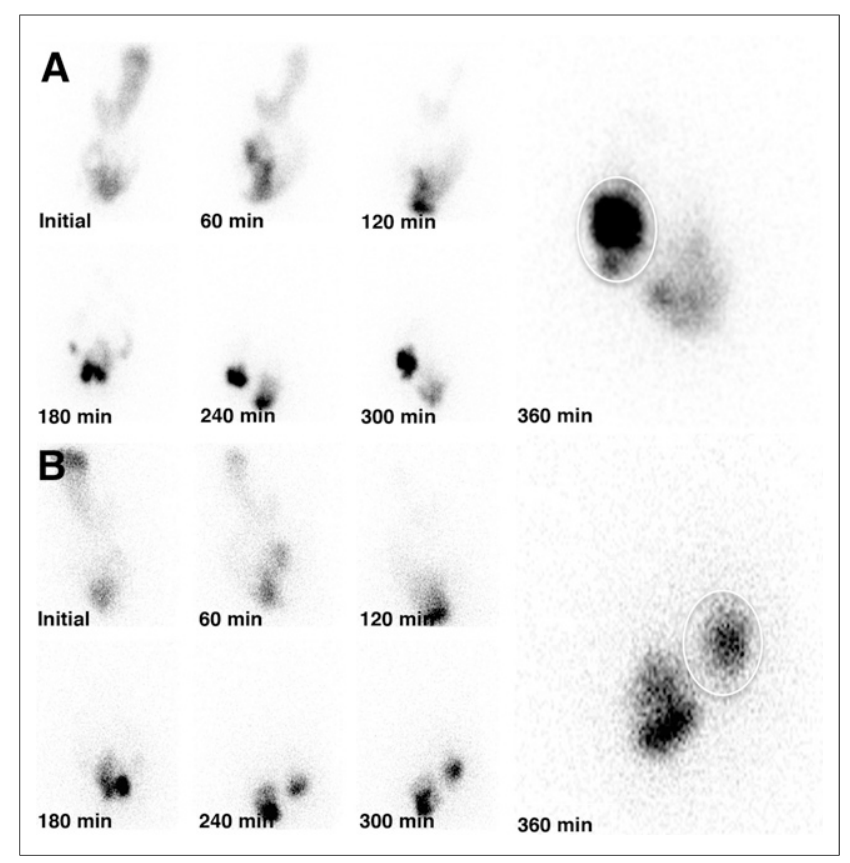

FIGURE 1. Normal small-bowel transit. Anterior (A) and posterior (B) views are shown, with ROI drawn around ileocecal value and cecum to calculate SBTI.

\section{Large-Bowel Transit}

For large-bowel transit, interobserver agreement for the GC was not significantly different for 24- and 48-h measurements (mean bias, 0.06-0.6) but was significantly different for $72 \mathrm{~h}$ (mean bias, 0.03-0.19; Pittman $P=0.03-0.21$ ). There was good correlation between the GC determination and the percentage colonic emptying at 24,48 , and $72 \mathrm{~h}\left(24-\mathrm{h} R^{2}=0.70 ; 48-\mathrm{h} R^{2}=0.90 ; 72-\mathrm{h}\right.$ $R^{2}=0.95$; all $\left.P<0.0001\right)$.

Using the 2- to 5-h and 6-h input, the counts to calculate percentage emptying were not significantly different $(P=$ 0.7820 ). The 6-h counts are now routinely used for clinical studies. Reference intestinal transit values are shown in Figures 1 and 2 .

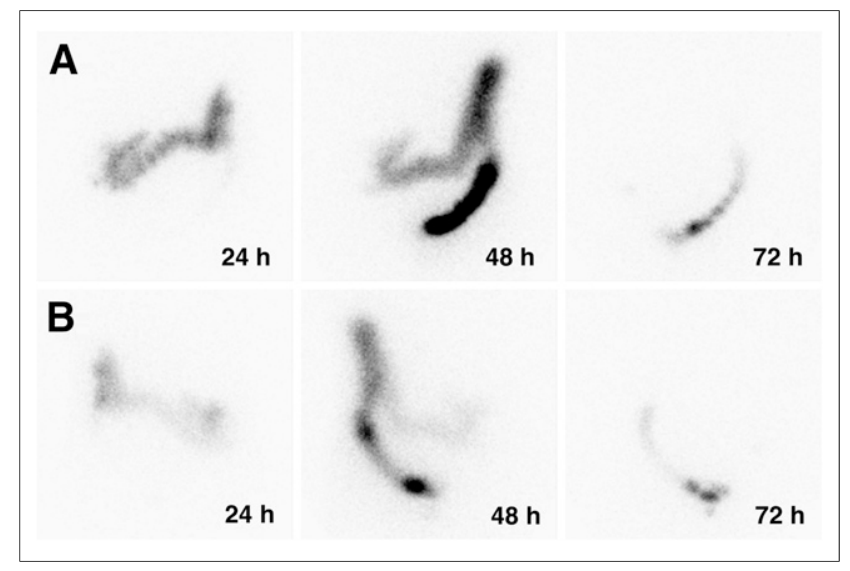

FIGURE 2. Normal large-bowel transit. Anterior (A) and posterior (B) views at 24,48 , and $72 \mathrm{~h}$ are shown. Most activity is in transverse colon by $24 \mathrm{~h}$, in descending colon by $48 \mathrm{~h}$, and in rectosigmoid colon or excreted by $72 \mathrm{~h}$. 


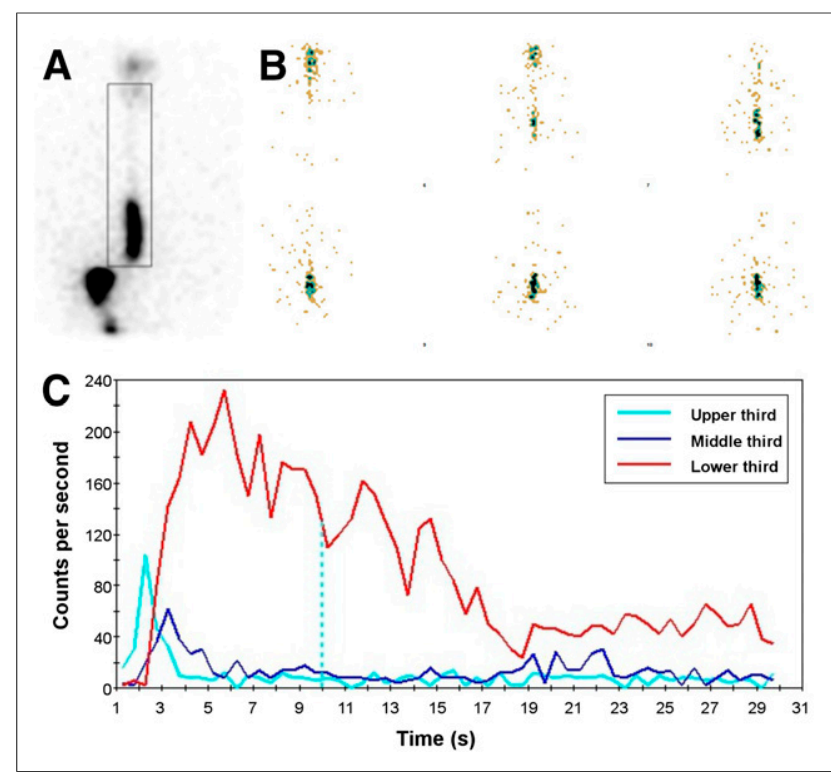

FIGURE 3. Delayed esophageal transit. (A) Summed image demonstrates delayed transit through lower third of esophagus. (B and $C$ ) Dynamic 0.5-s frames show rapid transit through first two thirds of esophagus in 1-2 $s$ but slow transit in distal third (B), confirmed by time-activity curve (red) (C). Esophageal transit time (ETT) was not reached by $30 \mathrm{~s}$. Percent esophageal emptying (\%EE) at $10 \mathrm{~s}$ was $54 \%$ (normal > 83\%).

\section{Clinical Results}

Of the first 18 patients, $72 \%$ (13/18) had abnormal transit in at least one gastrointestinal organ; $33 \%(6 / 18)$ had abnormal transit in more than one organ, $28 \%(5 / 18)$ had normal transit (Table 1). Examples of abnormal transit are shown in Figures 3-7. As a result, there was a subsequent change in diagnosis in $67 \%$ of patients $(12 / 18)$ and a change in management in 61\% (11/18).

\section{DISCUSSION}

In this paper, we have described our methodology for a scintigraphic comprehensive gastrointestinal transit study, reported reference values we determined from 18 healthy subjects, and related our initial clinical experience in 18 patients.

The methodology is unique in that it evaluates transit from esophagus to rectum, using ${ }^{111}$ In-DTPA for esophageal, liquid

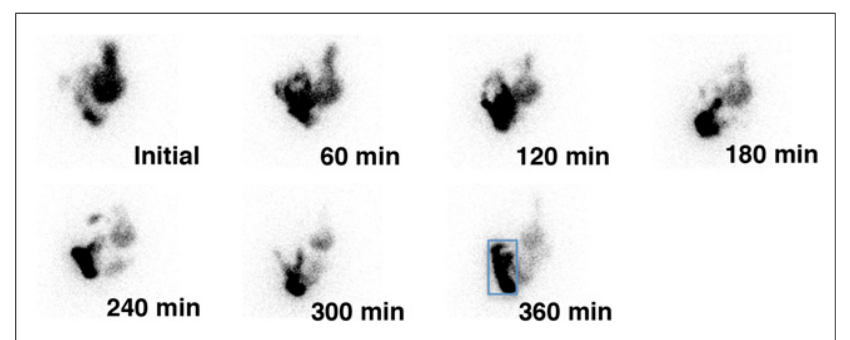

FIGURE 4. Small-bowel transit study corrected for delayed gastric transit. Patient had delayed gastric emptying in liquid-only study, liquid portion of combined liquid-solid study, and solid study. ${ }^{111}$ In counts in stomach are subtracted from total average counts between hours 2 and 5 , correcting small-bowel transit for gastric emptying. This patient had normal SBTI of $82 \%$ (normal $>50 \%$ ). Blue rectangle denotes ileocecal/ ascending colon ROI for calculation of SBTI.

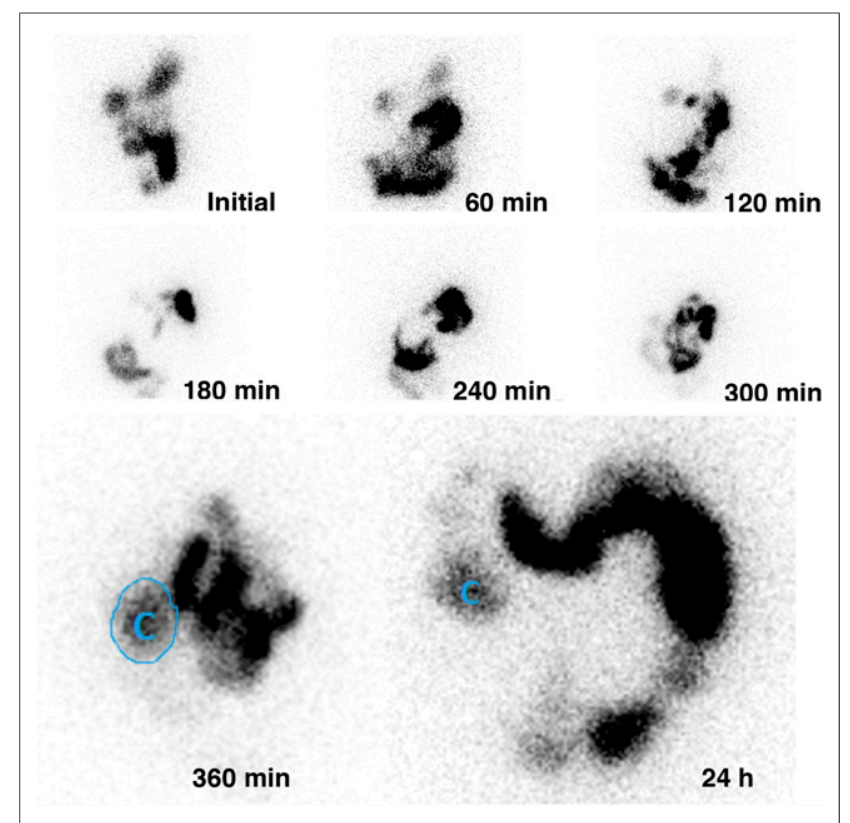

FIGURE 5. Delayed small-bowel transit. Anterior views are shown of patient with delayed SBTI (28\%). At $360 \mathrm{~min}, \mathrm{ROI}$ shows activity reaching cecum (C). Large-bowel emptying (84\%) is normal.

gastric, and small- and large-bowel transit, in conjunction with the standardized ${ }^{99 \mathrm{~m}} \mathrm{Tc}$-sulfur colloid-labeled solid meal and methodology recommended by the American Neurogastroenterology and Motility Society and the Society of Nuclear Medicine and Molecular Imaging, based on Tougas et al. (11-13).

Clinical indications for a comprehensive gastrointestinal transit study include suspected pandysmotility with overlapping regional symptoms, small-bowel dysmotility and therapy response for bacterial overgrowth, dysmotility in autoimmune (scleroderma) or other disorders (Ehlers-Danlos syndrome), and preoperative evaluation (achalasia, colonic inertia).

The first published study combining radionuclide gastric emptying and a small- and large-bowel transit study was in 1975 (14). Various methodologies and reference values have been described since $(15,16)$. To date, there is no standard methodology. Only a few institutions routinely perform "whole gut transit scintigraphy" (17,18). Our methodology differs from others previously described in several ways.

First, an esophageal transit study is performed. This initial 30-s study serves as a rapid screening test for delayed transit. It is a wellvalidated simple, short add-on to the comprehensive study. Numerous publications have shown it to have a high sensitivity as a stand-alone test for detecting esophageal transit disorders $(19,20)$. The method used is similar to methods previously described, and the reference values are consistent with previous literature (21-24).

The radionuclide methodology has been the gold standard for gastric-emptying evaluation for decades $(25,26)$. Before the published consensus recommendations, imaging clinics used various meals with different food content, often considerably higher in fat. In this comprehensive protocol, we have used the recommended standardized methodology and meal for the solid portion of the study, which is low in fat. The specific solid meal matters because it determines the rate of both solid and liquid emptying. The larger the meal, the greater the calories, carbohydrate, and fat consumed, and the slower the solid and liquid gastric emptying, 


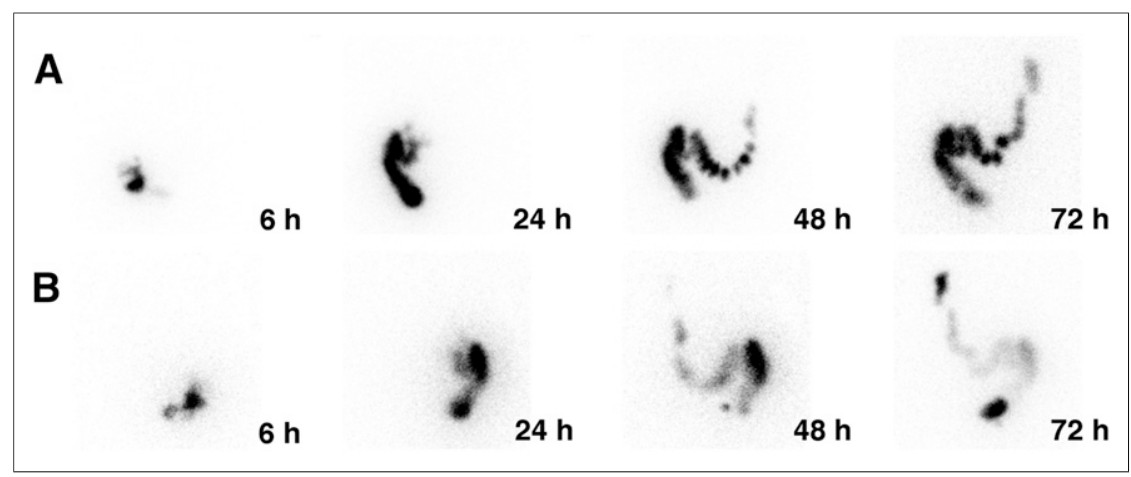

FIGURE 6. Delayed large-bowel transit. Anterior $(\mathrm{A})$ and posterior $(\mathrm{B})$ projection images show delayed ascending and transverse colonic transit. Little to no activity has transited into descending colon by $72 \mathrm{~h}$. limit of normal was $40 \%$ emptying at $1 \mathrm{~h}$ versus $50 \%$ published previously (7). One explanation may be that our reference values were determined using the consensus standardized solid meal (18).

Reference values for the standardized solid meal were not significantly different from those published by Tougas et al. (11). Even though our patients ingested a larger volume of water, $345 \mathrm{~mL}$ (esophageal, liquid only, and liquid-solid) compared with the $120 \mathrm{~mL}$ of water recommended, there was no effect on our solid-emptying results, consistent with previous reports that solid transit is not affected by the addition of liquid (30).

To quantify small-bowel transit, various and vice versa (27). To our knowledge, our comprehensive transit study is the first to publish and document reference values for liquid gastric emptying and small- and large-bowel transit using ${ }^{111}$ In-DTPA in conjunction with the standardized solid meal.

Two different dual-isotope dual-phase approaches to liquid gastric emptying have been described using ${ }^{111}$ In-DTPA in water and a ${ }^{99 m}$ Tc-labeled solid meal. One method begins with a 30-min liquid-only study followed by the solid study $(5,6)$. The other approach is to simultaneously administer the dual-isotope solid and liquid meal (7). Liquid-only studies have been shown to be more sensitive for detection of gastroparesis than the solid study using the sequential method (5-6), and both methods detect gastroparesis in $28 \%-32 \%$ of patients with normal solid emptying (5-7). The prior teaching was that liquid emptying is not delayed until after solid emptying becomes abnormal $(28,29)$.

In this comprehensive protocol, both methods were performed sequentially. We found that the liquid-only study emptied normally in a monoexponential pattern, whereas the combined liquid-solid study had a multiexponential clearance pattern. Liquid-only emptying had a shorter mean half-emptying time (14 vs. $39 \mathrm{~min}, P<$ 0.0001 ) than the liquid in the combined liquid-solid meal, raising the question of whether the two different methods measure the same physiology.

The reference liquid-emptying values for this liquid-solid study are different from those previously reported. The lower

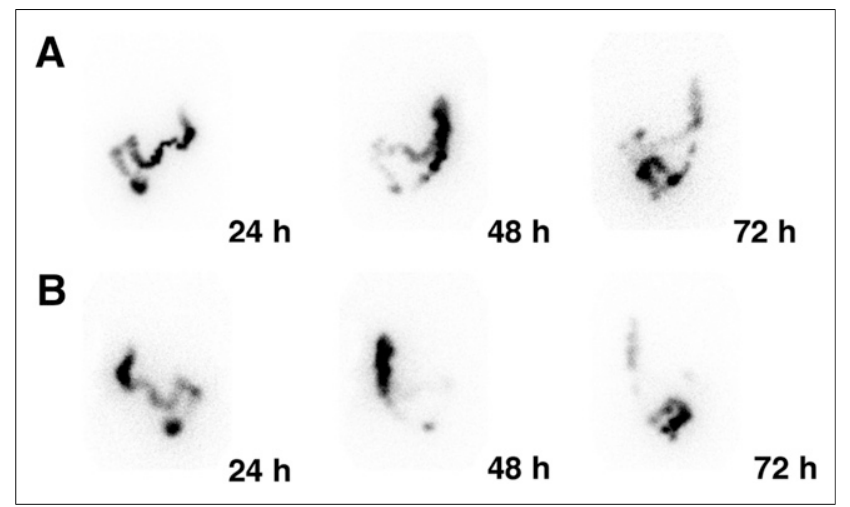

FIGURE 7. Delayed rectosigmoid transit. Anterior (A) and posterior (B) images show only $5 \%$ large-bowel transit by $72 \mathrm{~h}$, with most activity seen just proximal to mid-to-distal rectosigmoid colon. methods have been used. One approach has been to calculate orocecal transit time, that is, the time for radionuclide to accumulate in the cecum or the cumulative percentage entry into the colon over time $(18,31)$. This method has shown a generally good correlation with the hydrogen breath test (32). Another method has used SBTI, that is, the percentage activity that reaches the ileocecal value by $6 \mathrm{~h}$ (33). The latter method was chosen for this investigation. Our results differ from those that have been reported. We found normal small-bowel transit to be more than $50 \%$ at $6 \mathrm{~h}$; others using similar ${ }^{111}$ In methodology, but administering a whole-egg meal, reported reference values of more than $40 \%$ (28).

To investigate large-bowel transit, ${ }^{111}$ In-DTPA has been instilled directly into the proximal large bowel via cecal intubation (34). Although this is likely an accurate method, it is invasive and not practical clinically. Two different oral ingestion methodologies using different radiopharmaceuticals have been described. One has patients ingest a $\mathrm{pH}$-sensitive methacrylate-coated capsule containing ${ }^{111}$ In-labeled activated charcoal particles (17). The capsule dissolves at the $\mathrm{pH}$ of the large bowel (7.4), and the ${ }^{111} \mathrm{In}$ is released into the cecum.

An alternative method has been to administer the ${ }^{111}$ In-DTPA in water with a solid meal and follow its transit with imaging at various time points $(18,34)$. For colonic transit, the GC of activity has been used (28). We found this methodology demanding and time-consuming for technologists and physicians. Drawing multiple ROIs was not always reproducible.

Therefore, percentage colonic emptying at 24, 48, and $72 \mathrm{~h}$ was chosen. The 6-h counts were used as the initial counts. The entire field-of-view ROI measured percentage large-bowel emptying using commercially available gastric-emptying software. For interpretation, we first determine whether there is a quantitative delay in percentage emptying at any of 3 time points. We then provide additional qualitative visual assessment to distinguish between diffuse colonic delay and a regional abnormality, for example, the rectosigmoid colon. On the basis of the lower $95 \%$ confidence interval of calculated GC, our data show that healthy patients have most activity in or distal to the transverse colon by $24 \mathrm{~h}$, descending colon by $48 \mathrm{~h}$, and rectosigmoid colon by $72 \mathrm{~h}$. The percentage colonic-emptying method correlated well with the GC method.

The results in 18 patients suggest clinical value for our methodology for diagnosing various esophagogastrointestinal dysmotility disorders (Table 1). 


\section{CONCLUSION}

A radionuclide methodology and reference values have been described for a comprehensive clinical gastrointestinal transit study that includes esophageal transit, liquid and solid gastric emptying, small-bowel transit, and large-bowel transit, using ${ }^{111}$ In-DTPA in conjunction with the consensus-recommended standardized ${ }^{99 \mathrm{~m}} \mathrm{Tc}$-labeled solid meal. Our preliminary experience suggests its clinical value.

\section{DISCLOSURE}

The costs of publication of this article were defrayed in part by the payment of page charges. Therefore, and solely to indicate this fact, this article is hereby marked "advertisement" in accordance with 18 USC section 1734. No potential conflict of interest relevant to this article was reported.

\section{ACKNOWLEDGMENTS}

We acknowledge the hard work and dedication of the Johns Hopkins nuclear medicine technologists as we translated this project from research into clinical use.

\section{REFERENCES}

1. Tack J, Bisschops RAF, Sarnelli G. Pathophysiology and treatment of functional dyspepsia. Gastroenterology. 2004;127:1239-1255.

2. Thomforde GM, Camilleri M, Phillips SF, Forstrom LA. Evaluation of an inexpensive screening scintigraphic test of gastric emptying. J Nucl Med. 1995;36:93-96.

3. Guo J-P, Maurer AH, Fisher RS, Parkman HP. Extending gastric emptying scintigraphy from two to four hours detects more patients with gastroparesis. Dig Dis Sci. 2001;46:24-29.

4. Ziessman HA, Goetze S, Bonta D, Ravich W. Experience with a new standardized 4-hr gastric emptying protocol. J Nucl Med. 2007;48:568-572.

5. Ziessman HA, Okolo PI, Mullin GE, Chander A. Liquid gastric emptying is often abnormal when solid emptying is normal. J Clin Gastroenterol. 2009;43:639-643.

6. Ziessman HA, Chander A, Ramos A, Wahl RL, Clark JO. The added value of liquid gastric emptying compared to solid emptying alone. J Nucl Med. 2009; 50:726-731.

7. Sachdeva P, Malhotra N, Pathikonda M, et al. Gastric emptying of solids and liquids for evaluation for gastroparesis. Dig Dis Sci. 2011;56:1138-1146.

8. Lin HC, Prather C, Fisher RS, et al. Measurement of gastrointestinal transit. Dig Dis Sci. 2005;50:989-1004.

9. Szarka LA, Camilleri M. Methods for assessment of small-bowel and colonic transit. Semin Nucl Med. 2012;42:113-123.

10. Maurer AH, Camilleri M, Donohoe K, et al. The SNMMI and EANM practice guideline for small-bowel and colon transit 1.0. J Nucl Med. 2013;54:2004-2013.

11. Tougas G, Eaker EY, Abell TL, et al. Assessment of gastric emptying using a low fat meal: establishment of international control values. Am J Gastroenterol. 2000;95:1456-1462.
12. Abell TL, Camilleri M, Donohoe K, et al. Consensus recommendations for gastric emptying scintigraphy: a joint report of the American Neurogastroenterology and Motility Society and the Society of Nuclear Medicine. J Nucl Med Technol. 2008;36:44-54.

13. Abell TL, Camilleri M, Donohoe K, et al. Consensus recommendations for gastric emptying scintigraphy: a joint report of the American Neurogastroenterology and Motility Society and the Society of Nuclear Medicine. Am J Gastroenterol. 2008;103:753-763.

14. Waller SL. Differential measurement of small and large bowel transit times in constipation and diarrhea: a new approach. Gut. 1975;16:372-378.

15. Read NW, Al-Janabi MN, Holgate AM, et al. Simultaneous measurement of gastric emptying, small bowel residence and colonic filling of a solid meal by the use of the gamma camera. Gut. 1986;27:300-308.

16. Madsen JL, Jensen M. Gastrointestinal transit of technetium-99m-labeled cellulose fiber and indium-111-labeled plastic particles. J Nucl Med. 1989;30:402406.

17. Charles F, Camilleri M, Phillips SF, et al. Scintigraphy of the whole gut: clinical evaluation of transit disorders. Mayo Clin Proc. 1995;70:113-118.

18. Bonapace ES, Maurer AH, Davidoff S, et al. Whole gut transit scintigraphy in the clinical evaluation of patients with upper and lower gastrointestinal symptoms. Am J Gastroenterol. 2000;95:2838-2847.

19. Taillefer R, Jadliwalla M, Pellerin E, Lafontaine E, Duranceau A. Radionuclide transit study in detection of esophageal motor dysfunction: comparison with motility studies (manometry). J Nucl Med. 1990;31:1921-1926.

20. Stacey B, Patel P. Oesophageal scintigraphy for the investigation of dysphagia: in and out of favour-and underused when available. Eur J Nucl Med Mol Imaging. 2002;29:1216-1220.

21. Jørgensen F, Hesse B, Tromholt N, et al. Esophageal scintigraphy: reproducibility and normal ranges. J Nucl Med. 1992;33:2106-2109.

22. Tatsch K. Multiple swallow test for quantitative and qualitative evaluation of esophagal motility disorders. J Nucl Med. 1991;32:1365-1370.

23. Klein HA. Esophageal transit scintigraphy. Semin Nucl Med. 1995;25:306-317.

24. Mariani G, Boni GG, Barreca M, et al. Radionuclide gastroesophageal motor studies. J Nucl Med. 2004;45:1004-1028.

25. Griffith GH, Owen GM, Kirkman S, Shields R. Measurement of the rate of gastric emptying using chromium-51. Lancet. 1966;1:1244-1245.

26. Meyer JH, MacGregor IL, Gueller R, et al. ${ }^{99 \mathrm{~m}}$ Tc-tagged chicken liver as a marker of solid food in the human stomach. Am J Dig Dis. 1976;21:296304.

27. Collins PJ, Horowitz M, Cook DJ, et al. Gastric emptying in normal subjects: a reproducible technique using a single scintillation camera and computer system. Gut. 1983;24:1117-1125.

28. Parkman HP, Hasler WL, Fisher RS, et al. American Gastroenteological Association technical review on the diagnosis and treatment of gastroparesis. Gastroenterology. 2004;127:1592-1622.

29. Maurer AH, Parkman HP. Update on gastrointestinal scintigraphy. Semin Nucl Med. 2006;36:110-118.

30. Collins PJ, Houghton LA, Read NW, et al. Role of the proximal and distal stomach in mixed solid and liquid meal emptying. Gut. 1991;32:615-619.

31. von der Ohe MR, Camilleri M. Measurement of small bowel and colonic transit: indications and methods. Mayo Clin Proc. 1992;67:1169-1179.

32. Miller MA, Parkman HP, Urbain JC, et al. Comparison of scintigraphy and lactulose breath hydrogen test for assessment of orocecal transit. Dig Dis Sci. 1997;42:10-18.

33. Krevsky B, Maurer ASH, Niewiarowski T, et al. The effect of verapamil on human intestinal transit. Dig Dis Sci. 1992;37:919-924.

34. Notghi A, Hutchinson R, Kumar D, et al. Simplified method for measurement of segmental colonic transit time. Gut. 1994;35:976-981. 